\title{
(Research Article) \\ Minimization of Makespan in Job Shop Scheduling with Heuristic and Genetic Algorithms
}

\author{
Raushan Kumar ${ }^{1}$, Dr. Sridhar K. ${ }^{2^{*}}$ \\ ${ }^{1,2 *}$ Department of Mechanical Engineering, Chhatrapati Shivaji Institute of Technology Durg, Chhattisgarh, INDIA
}

\begin{abstract}
As the performance of manufacturing system is directly related to the optimum utilization of time and resources, optimal scheduling of work activities plays an important role. Job shop scheduling is an optimization problem, in which a set of jobs has to be processed on a set of machines such that some specific objective functions are to be minimized. Here an effort has been made to minimize the makespan and average job flow time of the job shop scheduling problem of following two natures: 5-job 3-machine problem and 10-job 5-machine problem. Data for these problems were collected from a small scale industry. This paper describes the steps in well-known heuristics such as Palmer's, Campbell Dudek and Smith (CDS) \& Nawaz Enscore and Ham (NEH) and a simple algorithm for proposed Genetic Algorithm. This paper investigates the present scheduling system followed by the industry and presents the solutions for the problems considered through heuristics and proposed Genetic Algorithm. Then the comparisons were made among the results obtained through proposed genetic algorithm, heuristics and LEKIN scheduling software to suggest an optimal sequence for the problems considered.
\end{abstract}

Keywords: Job shop scheduling, Makespan, Average job flow time, Heuristic algorithms, Genetic algorithm.

\section{Introduction}

Planning and scheduling are common to many different engineering domains, these are daily life activities. Whether the project is as large as the Magnificent Metro projects or something as simple as the redesign of the packaging for an industrial tape dispenser, both planning and scheduling play a vital role. Scheduling is an important decision making process that is used on regular basis in various manufacturing and service industries.Scheduling is the process of allocating the shared resources by applying the limiting factors of time and cost for the competing activities to perform a collection of tasks. A schedule is an allocation of operations to time intervals of the machines.All scheduling problems can be broadly categorized as, single-machine scheduling (only one machine) and multi-machine scheduling (more than one machine). Both job shop and flow shop production cope with a scheduling problem to find a feasible sequence of jobs on given machines such that certain objective(s) is to be optimized. These objectives can be minimization of makespan, minimization of average job flow time, minimization of maximum lateness, etc.

"Corresponding Author: e-mail: ksridhar@csitdurg.in, Contact No.$+91-8966000054$

ISSN 2320-7590

(C) 2018 Darshan Institute of Engg. \& Tech., All rights reserved
Typically, there are $m$ machines and $n$ jobs for scheduling. Jobs have to be processed on these machines with different routes or sequences. There are $n$ ! ways (solutions) to sequence jobs on each machine. For some type of scheduling problem, all machines use the similar sequence. So, the numbers of feasible solutions to find the optimal solution are $\mathrm{n}$ ! solutions. For the job shop scheduling problem, each machine has different sequences; therefore, number of feasible solution increase to $(\mathrm{n} !)^{\mathrm{m}}$ solutions.

Scheduling is easy and fast up to n-job and 2-machine scheduling problems, but it becomes NP-hard (nondeterministic polynomial-hard) when the number of machines $\geq 3$; that means when the size of problem grows up, the time for determining the optimal solutions of the problem increase exponentially. Therefore, the heuristic methods are used to speed up the process of finding the good enough optimal solution, where an exhaustive search is impractical. Heuristic refers to experience-based techniques for solving, learning, and discovery the new approaches to a problem.

The genetic algorithm is a search-based general optimization technique based on the principles of genetics and natural selection. It is an evolutionary algorithm inspired by Darwin's principle of natural selection. The central idea of natural selection is the survival of the fittest one for the next generation. What is the principle of natural selection? i.e. Select the Best, Discard the Rest; so the fittest will survive. 


\section{Literature Review}

In '50s, Johnson [1] first presented an algorithm for an njob 2-machine problem that can find the optimal sequence effectively without much calculation. Palmer's algorithm was given by Palmer [2] which used the slope index approach to sequence the jobs in optimal order with the decreasing value of slope index. This algorithm can be applied to n-job mmachine problems. A simple heuristic extension of Johnson's algorithm was proposed by Campbell et al. [3] popularly known as CDS heuristic algorithm. This uses the Johnson's algorithm in number of iteration before reaching the final solution. Nawaz et al. [4] developed a heuristic algorithm, based on the total processing time of the individual jobs. According to this algorithm, to obtain the optimal sequence priority should be given to the jobs having higher total processing time.

Man et al. [5] basically introduces the genetic algorithm (GA) as a complete entity, which can be used as design tool and also explain "why" and "when" GA should be used as an optimization tool. Laha and Mandal [6] have presented a new heuristic genetic algorithm (NGA) that combines the good features of both the genetic algorithms and heuristic search for the scheduling on makespan criterion in the flow shop environment. Li and Chen [7] did the study on characteristics of agile job shop scheduling problem and proposed a new genetic algorithm for solving the same. They improved the local optimal solution by adopting two-row chromosome structure based on working procedure and machine distribution. Verma et al. [8] developed an algorithm for job shop scheduling problem based on genetic algorithm and they proved that even a relatively simple genetic algorithm is capable for job shop scheduling. An overview of genetic algorithms was described for job shop scheduling problem [14]. Yadav and Prajapati [9] did the study of genetic algorithm and given an overview of genetic algorithm and modelling, its working and solution space.

Singhal et al. [10] proposed an improved algorithm which is obtained by modifying the NEH algorithm. They were shown that this algorithm produces improved quality solutions with algorithmic complexity same as the original algorithm.

Semanco and Modrak [11] proposed a constructive heuristic approach for the solution of the permutation flowshop problem, known as MOD heuristic. It was shown that the proposed algorithm is a feasible alternative for practical application when solving $\mathrm{n}$-job and $\mathrm{m}$-machine in flow-shop scheduling problems to give relatively good solutions in a short-time interval. A comparison was made among the various heuristics algorithms based on the make span by Modrak et al. [12]. The heuristics used are namely Palmer's, Gupta's algorithm, CDS, NEH and MOD heuristic algorithms. The NEH uses maximum iterations among all other while Palmer uses single iteration to reach the final result. Though Palmer's algorithm was very fast but it lacked accuracy and the result obtained from Palmer's algorithm don't coincide with the best result among all other heuristic algorithms.

Choudhari and Khanna [13] did the survey on various machine scheduling techniques for flow shop environment. Their objective was the minimization of total production time, while considering setup time, processing time, transporting time, returning time and they used the heuristic approach to arrive at the optimal solution. Silva and Klement [15] solved a multi-periods job shop scheduling problem using a generic and modular decision support tool. They demonstrated that the proposed tool can be easily adapted to several different planning or scheduling problems variants. Liu et al. [16] utilized the statistical measure skewness to construct a new priority rule, which is applied to the NEH heuristic, for solving scheduling problems in permutation flowshops.

\section{Methodology}

This section describes the various heuristic algorithms and proposed genetic algorithm which are used to arrive at the desired results.

3.1 Palmer's heuristic algorithm: This heuristic is based on the calculation of slope index for each job by assigning some specific weights to each machine. This heuristic comprises the following steps:

1. Consider an $n$-jobs $m$-machine problem.

2. Assign the specific weight $=\{m-(2 i-1)\}$ to the $i^{\text {th }}$ machine. Where $m$ is total number of machines.

3. Compute the slope $S_{j}$ for $j^{\text {th }}$ job as follows:

$$
S_{j}=-\sum_{i=1}^{m}\{m-(2 \times i-1)\} \times p_{i j}
$$

Where $m=$ number of machine in problem considered $\mathrm{p}_{\mathrm{ij}}=$ processing time of $\mathrm{j}^{\text {th }}$ job on $\mathrm{i}^{\text {th }}$ machine

4. Order the jobs in the sequence based on descending (decreasing) order of $S_{j}$ values.

5. Calculate the makespan for the sequence obtained.

3.2 Campbell Dudek Smith (CDS) heuristic algorithm: This algorithm converts a given n-job m-machine problem into $(\mathrm{m}-1)$ number of 2-machine surrogate problems. Each surrogate problem is solved using Johnson's rule. The value of makespan $\left(\mathrm{C}_{\max }\right)$ for each surrogate problem is found out.

The sequence of surrogate problem having minimum value of $\mathrm{C}_{\max }$ after applying Johnson's rule is selected for scheduling jobs on the machines. 
For each surrogate problem the processing time of all the jobs on both machines are calculated as follows:

$$
\begin{aligned}
& \mathrm{k}=\text { surrogate problem }=1,2,3, \ldots, \mathrm{m}-1 . \\
& \mathrm{j}=\mathrm{job}=1,2,3, \ldots, \mathrm{n} . \\
& \quad M_{1}^{\prime}=\sum_{i=1}^{k} p_{i j} \text { and } \quad M_{2}^{\prime}=\sum_{i=m-k+1}^{m} p_{i j}
\end{aligned}
$$

where $\mathrm{M} 1^{\prime}=$ processing time for the $1^{\text {st }}$ machine,

$$
\text { M2' = processing time for the } 2^{\text {nd }} \text { machine. }
$$

3.3 Nawaz Enscore Ham (NEH) heuristic algorithm: This algorithm constructs jobs sequence in iterative manner. NEH algorithm is described as:

1. Find the total work content $\left(T_{j}\right)$ using the following expression:

$$
T_{j}=\sum_{i=1}^{m} p_{i j}
$$

2. Arrange jobs in a work content list according to decreasing values of $T_{j}$.

3. Select first two jobs from the list, and form partial sequences by interchanging the place of the two jobs.

4. Out of the two partial sequences, select the partial sequence with lower value of $\mathrm{C}_{\max }$ and called it incumbent sequence.

5. Pick next job from the work content list, and place it all locations in the incumbent sequence. Calculate the value of $\mathrm{C}_{\max }$ for all the sequences.

6. Retain the sequence with minimum value of $\mathrm{C}_{\max }$ as incumbent sequence and discard all the other sequences.

7. If there is no job left in the work content list to be add to incumbent sequence, stop. Otherwise, go step 5.

\subsection{Proposed Genetic Algorithm:}

Table 1. Basic Terminologies in Genetic Algorithm

\begin{tabular}{|l|l|l|}
\hline \multicolumn{2}{|c|}{ Natural Evolution } & \multicolumn{1}{c|}{ Genetic Algorithm } \\
\hline 1 & Population & Solution (Set of Chromosomes) \\
\hline 2 & Chromosome & String (A Solution) \\
\hline 3 & Gene & Elemental Position in String \\
\hline 4 & Allele & Value at a certain Position \\
\hline 5 & Genotype & Coded String \\
\hline 6 & Phenotype & Uncoded String \\
\hline 7 & Fitness Function & Objective Function \\
\hline
\end{tabular}

Since the Genetic Algorithm is a general algorithm which applied to variety of problems and one can formulate it according to their problem and the complexity of the problem.
For the scheduling problems, 5-job 3-machine problem and 10-job 5-machine problem, following are the steps in the Proposed Genetic Algorithm based on the concepts of original one:

Step 1: Population Initialization: First step is to obtain the initial population, the set of solutions to proceed further. For scheduling problem, this is the job priority sequence with makespan and average job flow time. Set of 5 solutions is obtained through the combination of both solutions from the three heuristic algorithms and two solutions are randomly selected.

Step 2: Evaluation of Fitness: Fitness for each solution is determined using the fitness function. Multi-criteria approach has been used for fitness. Since our objective is to minimize the makespan and average job flow time, so the fitness function is: makespan and average job flow time. If first one is fail, next one will be used for fitness evaluation.

Step 3: Selection for Reproduction: This is the most important step to reach the optimal solution. For our scheduling problem fittest solution is selected on the basis of Rank Order Selection criteria. In this criterion, the solution having the least value for the fitness function is to be given the first rank and so on. The first ranked solution will be selected for reproduction.

Step 4: Genetic Operator: After the selection of solution, genetic operators are applied for the reproduction. There are two kinds of genetic operators: Crossover and Mutation. For the crossover two solutions are required and for mutation only one. Since there are scheduling problems for which mutation operator works better because solution is a sequence in which repetitions of job are not allowed. So shift mutation is used for reproduction. In the shift mutation genes (here jobs) are altered in positions through shifting from one position to another to obtain optimal sequence.

Step 5: New Population: New set of 5 solutions are obtained through mutation. Fitness is evaluated for each solution.

Step 6: Termination Criteria: If fitness value obtained in new population is better than fitness value of the initial population, then terminate the process; otherwise replace the least-fit value in previous set of solution by the fittest one from new population and repeat the procedure until termination condition is met. Perform at least three iterations, to obtain the good results. 


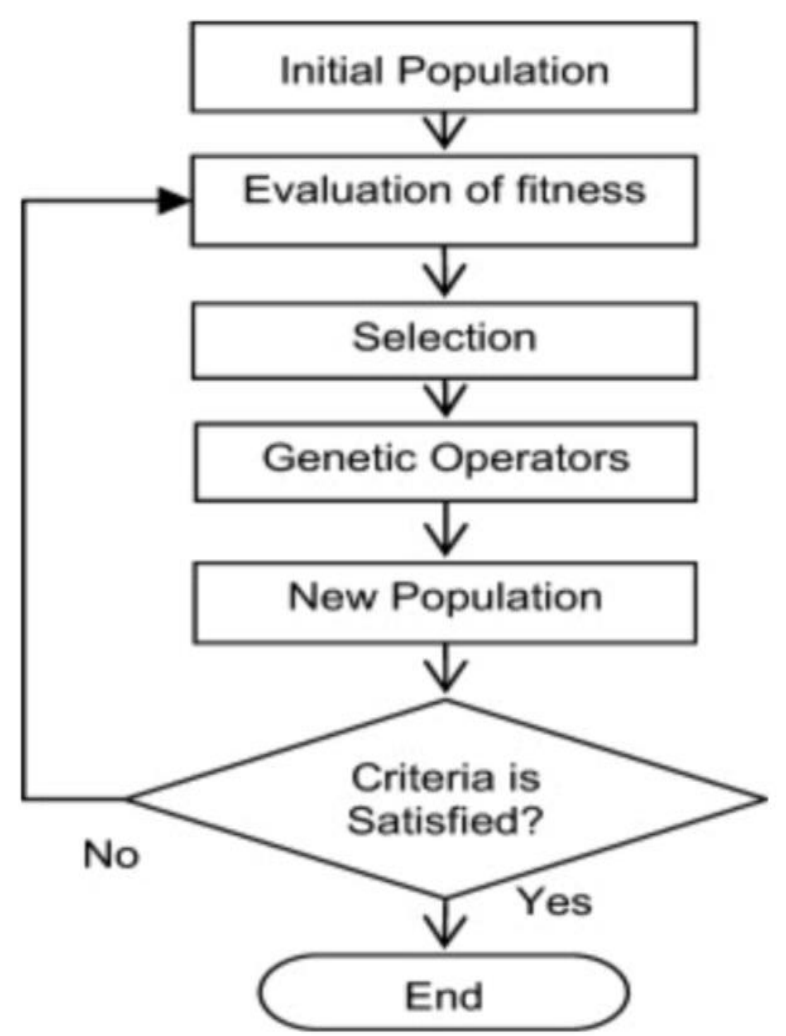

Figure 1. Flowchart of the Proposed Genetic Algorithm

\subsection{Pseudo code for the Proposed Genetic Algorithm:}

\{

initialize population; (population initialization based on heuristics and randomly chosen)

find the fitness of population; (fitness function is makespan)

while termination criteria not satisfied do (minimum makespan and average job flow time)

select fittest individual for mutation; (on the basis of rank order selection)

mutation to generate new population; (shift mutation)

calculation of fitness of each individual;

replace least fit individual in initial population with the fittest one of new population;

\} .

\section{Problem Description \& Solution}

The data collected for the scheduling problem are processing time, for the jobs on the machines in time unit of "minutes".

All the jobs are denoted $\mathrm{Jj}$, where $\mathrm{j}=1,2,3, \ldots ., \mathrm{n}$.

All the machines are denoted by Mi, where $\mathrm{i}=1,2,3, \ldots, \mathrm{m}$.
Table 2. 5-job 3-machine problem

\begin{tabular}{|c|c|c|c|}
\hline Job & M1 & M2 & M3 \\
\hline J1 & 10 & 10 & 12 \\
\hline J2 & 12 & 8 & 20 \\
\hline J3 & 16 & 6 & 14 \\
\hline J4 & 12 & 4 & 20 \\
\hline J5 & 20 & 8 & 8 \\
\hline
\end{tabular}

Table 3. 10-job 5-machine problem

\begin{tabular}{|c|c|c|c|c|c|}
\hline Job & M1 & M2 & M3 & M4 & M5 \\
\hline J1 & 32 & 8 & 8 & 28 & 24 \\
\hline J2 & 28 & 6 & 8 & 28 & 20 \\
\hline J3 & 36 & 10 & 10 & 30 & 26 \\
\hline J4 & 8 & 4 & 4 & 24 & 6 \\
\hline J5 & 8 & 4 & 4 & 24 & 4 \\
\hline J6 & 6 & 2 & 4 & 24 & 2 \\
\hline J7 & 4 & 4 & 4 & 22 & 4 \\
\hline J8 & 10 & 6 & 8 & 24 & 8 \\
\hline J9 & 12 & 8 & 6 & 24 & 6 \\
\hline J10 & 14 & 4 & 8 & 28 & 10 \\
\hline
\end{tabular}

Solution:

4.1 Through the scheduling technique followed by industry, i.e. First Come First Serve (FCFS) rule:

Table 4. Makespan calculation for J1,J2,J3,J4,J5

\begin{tabular}{|c|c|c|c|c|c|c|}
\hline \multirow{2}{*}{ Job } & \multicolumn{2}{|c|}{ M1 } & \multicolumn{2}{c|}{ M2 } & \multicolumn{2}{c|}{ M3 } \\
\cline { 2 - 7 } & In & Out & In & Out & In & Out \\
\hline J1 & 0 & 10 & 10 & 20 & 20 & 32 \\
\hline J2 & 10 & 22 & 22 & 30 & 32 & 52 \\
\hline J3 & 22 & 38 & 38 & 44 & 52 & 66 \\
\hline J4 & 38 & 50 & 50 & 54 & 66 & 86 \\
\hline J5 & 50 & 70 & 70 & 78 & 86 & 94 \\
\hline
\end{tabular}

$\mathrm{C}_{\max }=94$ minutes.

$\mathrm{T}_{\mathrm{avg}}=(32+52+66+66+94) / 5=66$ minutes.

Table 5. Makespan calculation for J1,J2,J3,J4,J5,J6,J7,J8,J9,J10

\begin{tabular}{|c|c|c|c|c|c|c|c|c|c|c|}
\hline \multirow{2}{*}{ Job } & \multicolumn{2}{|c|}{ M1 } & \multicolumn{2}{|c|}{ M2 } & \multicolumn{2}{|c|}{ M3 } & \multicolumn{2}{|c|}{ M4 } & \multicolumn{2}{|c|}{ M5 } \\
\cline { 2 - 12 } & In & Out & In & Out & In & Out & In & Out & In & Out \\
\hline \multirow{2}{*}{ J1 } & 0 & 32 & 32 & 40 & 40 & 48 & 48 & 76 & 76 & 100 \\
\hline J2 & 32 & 60 & 60 & 66 & 66 & 74 & 76 & 104 & 104 & 124 \\
\hline J3 & 60 & 96 & 96 & 106 & 106 & 116 & 116 & 146 & 146 & 172 \\
\hline J4 & 96 & 104 & 106 & 110 & 116 & 120 & 146 & 170 & 172 & 178 \\
\hline J5 & 104 & 112 & 112 & 116 & 120 & 124 & 170 & 194 & 194 & 198 \\
\hline J6 & 112 & 118 & 118 & 120 & 124 & 128 & 194 & 218 & 218 & 220 \\
\hline J7 & 118 & 122 & 122 & 126 & 128 & 132 & 218 & 240 & 240 & 244 \\
\hline J8 & 122 & 132 & 132 & 138 & 138 & 146 & 240 & 264 & 264 & 272 \\
\hline J9 & 132 & 144 & 144 & 152 & 152 & 158 & 264 & 288 & 288 & 294 \\
\hline J10 & 144 & 158 & 158 & 162 & 162 & 170 & 288 & 316 & 316 & 326 \\
\hline
\end{tabular}

$\mathrm{C}_{\max }=326$ minutes $\& \mathrm{~T}_{\mathrm{avg}}=(100+124+172+178+198+$ $220+244+272+294+326) / 1=212.8$ minutes. 
4.2 Through Palmer's Heuristic Algorithm: According to this algorithm slope index for each job is calculated using (1).

Table 6. Slope index calculation in 5-job 3-machine problem

\begin{tabular}{|c|c|c|c|c|}
\hline Weight & 2 & 0 & -2 & \\
\hline Job & M1 & M2 & M3 & S $_{\mathrm{j}}$ \\
\hline J1 & 10 & 10 & 12 & 4 \\
\hline J2 & 12 & 8 & 20 & 16 \\
\hline J3 & 16 & 6 & 14 & -4 \\
\hline J4 & 12 & 4 & 20 & 16 \\
\hline J5 & 20 & 8 & 8 & -24 \\
\hline
\end{tabular}

Two sequences are possible: J2,J4,J1,J3,J5 and $\mathrm{J} 4, \mathrm{~J} 2, \mathrm{~J} 1, \mathrm{~J} 3, \mathrm{~J} 5$ out of which latter one gives best result.

Table 7. Makespan calculation for J4,J2,J1,J3,J5

\begin{tabular}{|c|c|c|c|c|c|c|}
\hline \multirow{2}{*}{ Job } & \multicolumn{2}{|c|}{ M1 } & \multicolumn{2}{c|}{ M2 } & \multicolumn{2}{c|}{ M3 } \\
\cline { 2 - 7 } & In & Out & In & Out & In & Out \\
\hline J4 & 0 & 12 & 12 & 16 & 12 & 36 \\
\hline J2 & 12 & 24 & 24 & 32 & 36 & 56 \\
\hline J1 & 24 & 34 & 34 & 44 & 56 & 68 \\
\hline J3 & 34 & 50 & 50 & 56 & 68 & 82 \\
\hline J5 & 50 & 70 & 70 & 78 & 82 & 90 \\
\hline
\end{tabular}

$\mathrm{C}_{\max }=90$ minutes \& $\mathrm{T}_{\mathrm{avg}}=(36+56+68+82+90) / 5=66.4$ minutes.

For the 10-job 5-machine problem, after arranging jobs in order with decreasing value of $\mathrm{Sj}$ value gives optimal sequence as J7,J4,J10,J6,J8,J5,J2,J9,J1,J3.

Table 8. Makespan calculation for J7,J4,J10,J6,J8,J5,J2,J9,J1,J3

\begin{tabular}{|c|c|c|c|c|c|c|c|c|c|c|}
\hline \multirow{2}{*}{ Job } & \multicolumn{2}{|c|}{ M1 } & \multicolumn{2}{|c|}{ M2 } & \multicolumn{2}{c|}{ M3 } & \multicolumn{2}{|c|}{ M4 } & \multicolumn{2}{c|}{ M5 } \\
\cline { 2 - 11 } & In & Out & In & Out & In & Out & In & Out & In & Out \\
\hline J7 & 0 & 4 & 4 & 8 & 8 & 12 & 12 & 34 & 34 & 38 \\
\hline J4 & 4 & 12 & 12 & 16 & 16 & 20 & 34 & 58 & 58 & 64 \\
\hline J10 & 12 & 26 & 26 & 30 & 30 & 38 & 58 & 86 & 86 & 96 \\
\hline J6 & 26 & 32 & 32 & 34 & 38 & 42 & 86 & 110 & 110 & 112 \\
\hline J8 & 32 & 42 & 42 & 48 & 48 & 56 & 110 & 134 & 134 & 142 \\
\hline J5 & 42 & 50 & 50 & 54 & 56 & 60 & 134 & 158 & 158 & 162 \\
\hline J2 & 50 & 78 & 78 & 84 & 84 & 92 & 158 & 186 & 186 & 206 \\
\hline J9 & 78 & 90 & 90 & 98 & 98 & 104 & 186 & 210 & 210 & 216 \\
\hline J1 & 90 & 122 & 122 & 130 & 130 & 138 & 210 & 238 & 238 & 262 \\
\hline J3 & 122 & 158 & 158 & 168 & 168 & 178 & 238 & 268 & 268 & 294 \\
\hline
\end{tabular}

$\mathrm{C}_{\max }=294$ minutes \& $\mathrm{T}_{\mathrm{avg}}=(38+64+96+112+142+162+$ $206+216+262+294) / 10=159.2$ minutes.
4.3 Through CDS Heuristic Algorithm: Makespan calculations for the optimal sequences obtained through CDS algorithm are:

Table 9. Makespan calculation for J4,J1,J2,J3,J5

\begin{tabular}{|c|c|c|c|c|c|c|}
\hline \multirow{2}{*}{ Job } & \multicolumn{2}{|c|}{ M1 } & \multicolumn{2}{c|}{ M2 } & \multicolumn{2}{c|}{ M3 } \\
\cline { 2 - 7 } & In & Out & In & Out & In & Out \\
\hline J4 & 0 & 12 & 12 & 16 & 16 & 36 \\
\hline J1 & 12 & 22 & 22 & 32 & 36 & 48 \\
\hline J2 & 22 & 34 & 34 & 42 & 48 & 68 \\
\hline J3 & 34 & 50 & 50 & 56 & 68 & 82 \\
\hline J5 & 50 & 70 & 70 & 78 & 82 & 90 \\
\hline
\end{tabular}

$\mathrm{C}_{\max }=90$ minutes $\& \mathrm{~T}_{\max }=(36+48+68+82+90) / 5=64.8$ minutes.

Table 10. Makespan calculation for J7,J4,J5,J9,J3,J1,J2,J10,J8,J6

\begin{tabular}{|c|c|c|c|c|c|c|c|c|c|c|}
\hline \multirow{2}{*}{ Job } & \multicolumn{2}{|c|}{ M1 } & \multicolumn{2}{|c|}{ M2 } & \multicolumn{2}{|c|}{ M3 } & \multicolumn{2}{|c|}{ M4 } & \multicolumn{2}{|c|}{ M5 } \\
\cline { 2 - 11 } & In & Out & In & Out & In & Out & In & Out & In & Out \\
\hline J7 & 1 & 4 & 4 & 8 & 8 & 12 & 12 & 34 & 34 & 38 \\
\hline J4 & 4 & 12 & 12 & 16 & 16 & 20 & 34 & 58 & 58 & 64 \\
\hline J5 & 12 & 20 & 20 & 24 & 24 & 28 & 58 & 82 & 82 & 86 \\
\hline J9 & 20 & 32 & 32 & 40 & 40 & 46 & 82 & 106 & 106 & 112 \\
\hline J3 & 32 & 68 & 68 & 78 & 78 & 88 & 106 & 136 & 136 & 162 \\
\hline J1 & 68 & 100 & 100 & 108 & 108 & 116 & 136 & 164 & 164 & 188 \\
\hline J2 & 100 & 128 & 128 & 134 & 134 & 142 & 164 & 192 & 192 & 212 \\
\hline J10 & 128 & 142 & 142 & 146 & 146 & 154 & 192 & 220 & 220 & 230 \\
\hline J8 & 142 & 152 & 152 & 158 & 158 & 164 & 220 & 244 & 244 & 252 \\
\hline J6 & 152 & 158 & 158 & 160 & 164 & 168 & 244 & 268 & 268 & 270 \\
\hline
\end{tabular}

$\mathrm{C}_{\max }=270$ minutes $\& \mathrm{~T}_{\max }=(38+64+86+112+162+188+$ $212+230+252+270) / 10=161.4$ minutes.

4.4 Through NEH Heuristic Algorithm: According to this algorithm total work content for each job is calculated using (3) and then jobs are sequenced in order with decreasing value of total work content to obtain partial sequence. After performing all iterations, the makespan calculation for optimal sequences through this algorithm are:

Table 11. Makespan calculation for J4,J1,J3,J2,J5

\begin{tabular}{|c|c|c|c|c|c|c|}
\hline \multirow{2}{*}{ Job } & \multicolumn{2}{|c|}{ M1 } & \multicolumn{2}{c|}{ M2 } & \multicolumn{2}{|c|}{ M3 } \\
\cline { 2 - 7 } & In & Out & In & Out & In & Out \\
\hline J4 & 0 & 12 & 12 & 16 & 16 & 36 \\
\hline J1 & 12 & 22 & 22 & 32 & 36 & 48 \\
\hline J3 & 22 & 38 & 38 & 44 & 48 & 62 \\
\hline J2 & 38 & 50 & 50 & 58 & 62 & 82 \\
\hline J5 & 50 & 70 & 70 & 78 & 82 & 90 \\
\hline
\end{tabular}


$\mathrm{C}_{\max }=90$ minutes $\& \mathrm{~T}_{\mathrm{avg}}=(36+48+62+82+90) / 5=63.6$ minutes.

Table 12. Makespan calculation for J7,J4,J5,J9,J8,J10,J3,J1,J2,J6

\begin{tabular}{|c|c|c|c|c|c|c|c|c|c|c|}
\hline \multirow{2}{*}{ Job } & \multicolumn{2}{|c|}{ M1 } & \multicolumn{2}{|c|}{ M2 } & \multicolumn{2}{|c}{ M3 } & \multicolumn{2}{|c|}{ M4 } & \multicolumn{2}{|c|}{ M5 } \\
\cline { 2 - 11 } & In & Out & In & Out & In & Out & In & Out & In & Out \\
\hline J7 & 0 & 4 & 4 & 8 & 8 & 12 & 12 & 34 & 34 & 38 \\
\hline J4 & 4 & 12 & 12 & 16 & 16 & 20 & 34 & 58 & 58 & 64 \\
\hline J5 & 12 & 20 & 20 & 24 & 24 & 28 & 58 & 82 & 82 & 86 \\
\hline J9 & 20 & 32 & 32 & 40 & 40 & 46 & 82 & 106 & 106 & 112 \\
\hline J8 & 32 & 42 & 42 & 48 & 48 & 56 & 106 & 130 & 130 & 138 \\
\hline J10 & 42 & 56 & 56 & 60 & 60 & 68 & 130 & 158 & 158 & 168 \\
\hline J3 & 56 & 92 & 92 & 102 & 102 & 112 & 158 & 188 & 188 & 214 \\
\hline J1 & 92 & 124 & 124 & 132 & 132 & 140 & 188 & 216 & 216 & 240 \\
\hline J2 & 124 & 152 & 152 & 158 & 158 & 166 & 216 & 244 & 244 & 264 \\
\hline J6 & 152 & 158 & 158 & 160 & 166 & 170 & 244 & 268 & 268 & 270 \\
\hline
\end{tabular}

$\mathrm{C}_{\max }=270$ minutes $\& \mathrm{~T}_{\mathrm{avg}}=(38+64+86+112+138+168+$ $214+240+264+270) / 10=159.4$ minutes.

\subsection{Through Proposed Genetic Algorithm:}

\subsubsection{For 5-job 3-machine problem:}

Table 13. Initial population with their fitness value

\begin{tabular}{|c|c|c|c|c|}
\hline Member & From & Sequence & $\mathrm{C}_{\max }$ & $\mathrm{T}_{\text {avg }}$ \\
\hline 1 & Palmer's heuristic & J4,J2,J1,J3,J5 & 90 & 66.4 \\
\hline 2 & CDS heuristic & J4,J1,J2,J3,J5 & 90 & 64.8 \\
\hline 3 & NEH heuristic & J4,J1,J3,J2,J5 & 90 & 63.6 \\
\hline 4 & Randomly chosen & J1,J4,J2,J5,J3 & 94 & 66 \\
\hline 5 & Randomly chosen & J2,J5,J3,J4,J1 & 100 & 68.8 \\
\hline
\end{tabular}

After performing all three iterations of the proposed genetic algorithm optimal sequence was obtained as J4,J3,J1,J2,J5.

Table 14. Makespan calculation for $\mathrm{J} 4, \mathrm{~J} 3, \mathrm{~J} 1, \mathrm{~J} 2, \mathrm{~J} 5$

\begin{tabular}{|c|c|c|c|c|c|c|}
\hline \multirow{2}{*}{ Job } & \multicolumn{2}{|c|}{ M1 } & \multicolumn{2}{c|}{ M2 } & \multicolumn{2}{c|}{ M3 } \\
\cline { 2 - 7 } & In & Out & In & Out & In & Out \\
\hline J4 & 0 & 12 & 12 & 16 & 16 & 36 \\
\hline J3 & 12 & 28 & 28 & 34 & 36 & 50 \\
\hline J1 & 28 & 38 & 38 & 48 & 50 & 62 \\
\hline J2 & 38 & 50 & 50 & 58 & 62 & 82 \\
\hline J5 & 50 & 70 & 70 & 78 & 82 & 90 \\
\hline
\end{tabular}

$\mathrm{C}_{\max }=90$ minutes \& $\mathrm{T}_{\mathrm{avg}}=(36+50+62+82+90) / 5=64$ minutes.

\subsubsection{For 10-job 5-machine problem:}

Table 15. Initial population with their fitness value

\begin{tabular}{|c|c|c|c|c|}
\hline Member & From & Sequence & $\mathrm{C}_{\max }$ & $\mathrm{T}_{\text {avg }}$ \\
\hline 1 & $\begin{array}{c}\text { Palmer's } \\
\text { heuristic }\end{array}$ & J7,J4,J10,J6,J8,J5,J2,J9,J1,J3 & 294 & 159.2 \\
\hline 2 & $\begin{array}{c}\text { CDS } \\
\text { heuristic }\end{array}$ & J7,J4,J5,J9,J3,J1,J2,J10,J8,J6 & 270 & 161.4 \\
\hline 3 & $\begin{array}{c}\text { NEH } \\
\text { heuristic }\end{array}$ & J7,J4,J5,J9,J8,J10,J3,J1,J2,J6 & 270 & 159.4 \\
\hline 4 & $\begin{array}{c}\text { Randomly } \\
\text { chosen }\end{array}$ & J6,J7,J5,J4,J8,J9,J10,J2,J1,J3 & 294 & 157.4 \\
\hline 5 & $\begin{array}{c}\text { Randomly } \\
\text { chosen }\end{array}$ & J6,J7,J4,J5,J8,J10,J9,J2,J1,J3 & 288 & 156.8 \\
\hline
\end{tabular}

After performing all three iterations of the proposed genetic algorithm optimal sequence was obtained as J7,J4,J5,J9,J8,J2,J1,J3,J10,J6.

Table 16. Makespan calculation for J7,J4,J5,J9,J8,J2,J1,J3,J10,J6

\begin{tabular}{|c|c|c|c|c|c|c|c|c|c|c|}
\hline \multirow{2}{*}{ Job } & \multicolumn{2}{|c|}{ M1 } & \multicolumn{2}{|c|}{ M2 } & \multicolumn{2}{|c|}{ M3 } & \multicolumn{2}{|c|}{ M4 } & \multicolumn{2}{|c|}{ M5 } \\
\cline { 2 - 12 } & In & Out & In & Out & In & Out & In & Out & In & Out \\
\hline J7 & 0 & 4 & 4 & 8 & 8 & 12 & 12 & 34 & 34 & 38 \\
\hline J4 & 4 & 12 & 12 & 16 & 16 & 20 & 34 & 58 & 58 & 64 \\
\hline J5 & 12 & 20 & 20 & 24 & 24 & 28 & 58 & 82 & 82 & 86 \\
\hline J9 & 20 & 32 & 32 & 40 & 40 & 46 & 82 & 106 & 106 & 112 \\
\hline J8 & 32 & 42 & 42 & 48 & 48 & 56 & 106 & 130 & 130 & 138 \\
\hline J2 & 42 & 70 & 70 & 76 & 76 & 84 & 130 & 158 & 158 & 178 \\
\hline J1 & 70 & 102 & 102 & 110 & 110 & 118 & 158 & 186 & 186 & 210 \\
\hline J3 & 102 & 138 & 138 & 148 & 148 & 158 & 186 & 216 & 216 & 242 \\
\hline J10 & 138 & 152 & 152 & 156 & 158 & 166 & 216 & 244 & 244 & 254 \\
\hline J6 & 152 & 158 & 158 & 160 & 166 & 170 & 244 & 268 & 268 & 270 \\
\hline
\end{tabular}

$\mathrm{C}_{\max }=270$ minutes $\& \mathrm{~T}_{\mathrm{avg}}=(38+64+86+112+138+178+$ $210+242+254+270) / 10=159.2$ minutes.

\subsection{Through LEKIN Scheduling Software:}

\subsubsection{For 5-job 3-machine problem:}
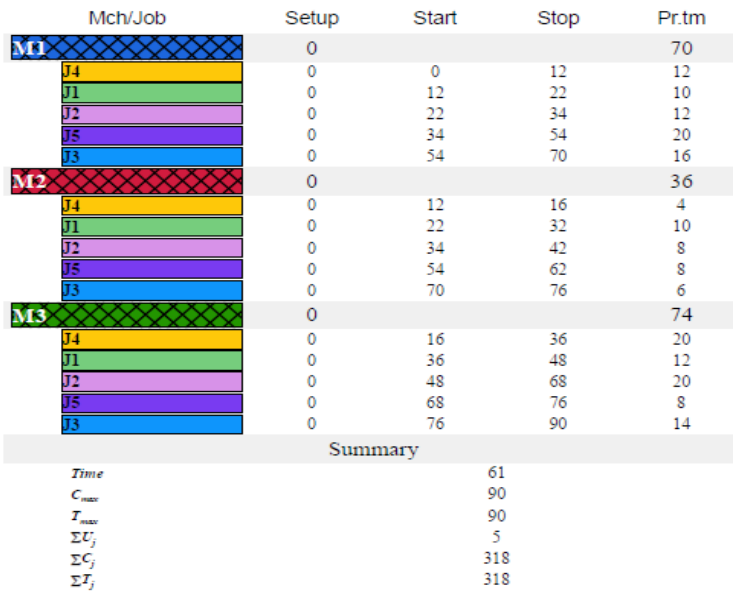

Figure 2. Makespan calculation through LEKIN software

$\mathrm{C}_{\max }=90$ minutes $\& \mathrm{~T}_{\mathrm{avg}}=318 / 5=63.6$ minutes. 


\subsubsection{For 10-job 5-machine problem:}

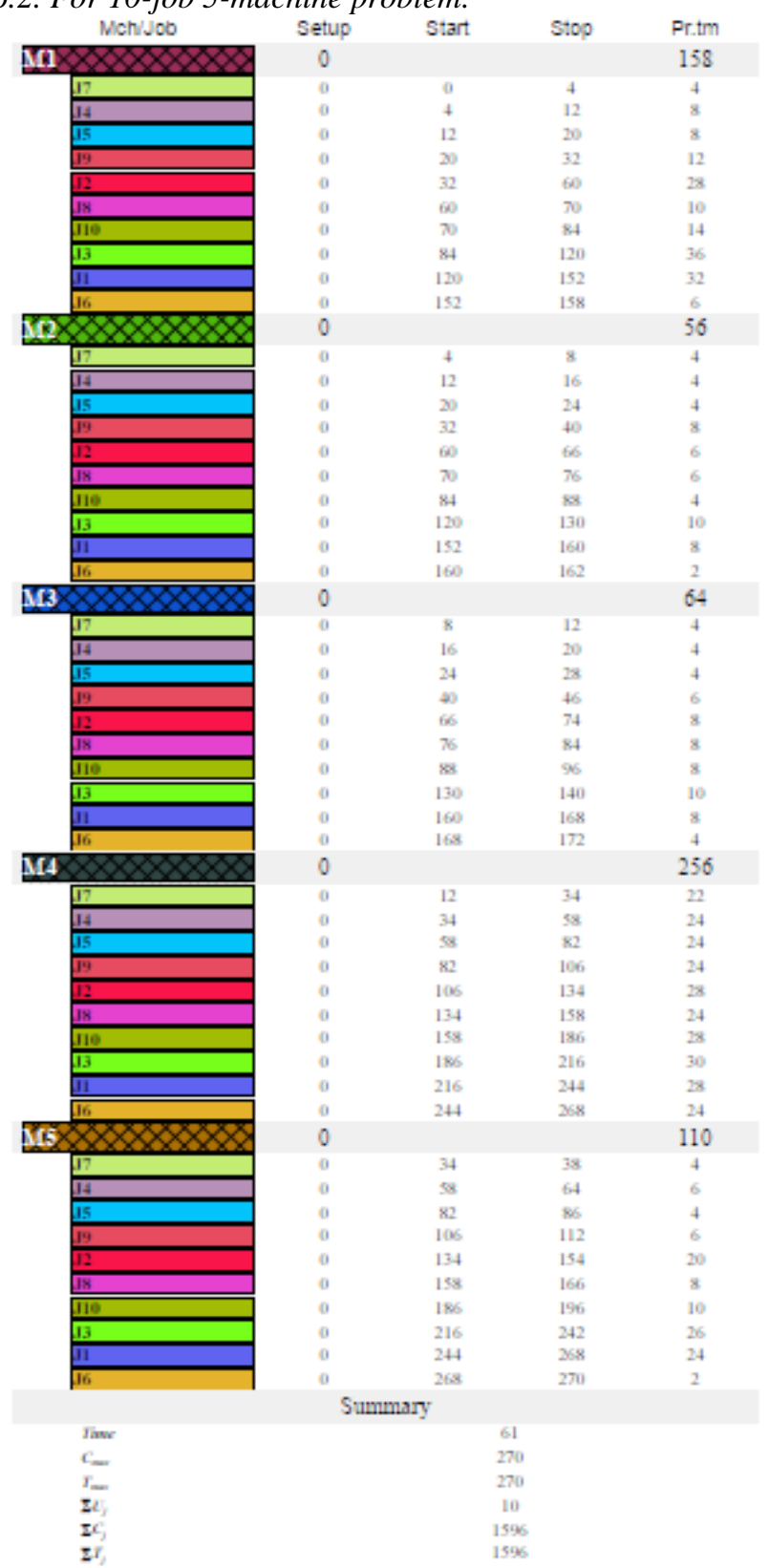

Figure 3. Makespan calculation through LEKIN software

$\mathrm{C}_{\max }=270$ minutes $\& \mathrm{~T}_{\text {avg }}=1596 / 10=159.6$ minutes.

\section{Results Analysis}

Table 17. Results for 5-job 3-machine problem

\begin{tabular}{|c|c|c|c|}
\hline Algorithm & Sequence & $\mathrm{C}_{\max }$ & $\mathrm{T}_{\text {avg }}$ \\
\hline FCFS Rule & $\mathrm{J} 1, \mathrm{~J} 2, \mathrm{~J} 3, \mathrm{~J} 4, \mathrm{~J} 5$ & 94 & 66 \\
\hline Palmer & J4,J2,J1,J3,J5 & 90 & 66.4 \\
\hline CDS & J4,J1,J2,J3,J5 & 90 & 64.8 \\
\hline NEH & J4,J1,J3,J2,J5 & 90 & 63.6 \\
\hline Proposed GA & J4,J3,J1,J2,J5 & 90 & 64 \\
\hline LEKIN Software & J4,J1,J2,J5,J3 & 90 & 63.6 \\
\hline
\end{tabular}

Table 18. Results for 10-job 5-machine problem

\begin{tabular}{|c|c|c|c|}
\hline Algorithm & Sequence & $\mathrm{C}_{\max }$ & $\mathrm{T}_{\text {avg }}$ \\
\hline FCFS Rule & J1,J2,J3,J4,J5,J6,J7,J8,J9,J10 & 326 & 212.8 \\
\hline Palmer & J7,J4,J10,J6,J8,J5,J2,J9,J1,J3 & 294 & 159.2 \\
\hline CDS & J7,J4,J5,J9,J3,J1,J2,J10,J8,J6 & 270 & 161.4 \\
\hline NEH & J7,J4,J5,J9,J8,J10,J3,J1,J2,J6 & 270 & 159.4 \\
\hline Proposed GA & J7,J4,J5,J9,J8,J2,J1,J3,J10,J6 & 270 & 159.2 \\
\hline $\begin{array}{c}\text { LEKIN } \\
\text { Software }\end{array}$ & J7,J4,J5,J9,J2,J8,J10,J3,J1,J6 & 270 & 159.6 \\
\hline
\end{tabular}

\section{Conclusions}

The investigations of the results obtained, allows to be made some important conclusions as follows:

- The scheduling sequences of jobs through the industry's scheduling system are far away from the optimal one.

- For 5-job 3-machine problem NEH heuristic gives the best results among the heuristic algorithms, this is also the optimal solution for this problem. Results from the LEKIN software are also same but with different sequence. The results for this problem through the Proposed Genetic Algorithm are very close to the optimal solution.

- Optimal sequence for this problem is $\mathrm{J} 4, \mathrm{~J} 1, \mathrm{~J} 3, \mathrm{~J} 2, \mathrm{~J} 5$ with makespan of 90 minutes and average job flow time of 63.6 minutes.

- For 10-job 5-machine problem NEH heuristic gives the best results among the heuristic algorithms, whereas optimal solutions were obtained through the Proposed Genetic Algorithm. The results of the LEKIN software are agreed with results of the Proposed Genetic Algorithm with same makespan and very small difference in average job flow time, although there is different sequence obtained from both.

- Optimal sequence for this problem is J7,J4,J5,J9,J8,J2,J1,J3,J10,J6 with makespan of 270 minutes and average job flow time of 159.2 minutes.

- For 5-job 3-machine problem the Proposed GA gives the different job priority sequence with same makespan, as per the optimal sequence obtained from the NEH heuristic algorithm. For 10-job 5machine problem the Proposed GA gives the optimal job priority sequence with least average job flow time of 159.2 minutes for minimum makespan of 270 minutes. When compared with the solutions of other heuristic algorithms, the results obtained through the Proposed GA are optimal with respect to minimum makespan and minimum average job flow time.

Since the Proposed Genetic Algorithm is formulated especially for the problems considered according to the 
complexity and circumstances of the problems with some assumptions, so it cannot be generalized for all kind of scheduling problem. Proposed Genetic Algorithm is very simple in nature and easy to implement.

Proposed Genetic Algorithm can execute the optimal solution in short time compared with heuristic algorithms. Proposed GA can give optimal solution compared with heuristic algorithms. But nature of job shop problems are not similar, so tailor made GA algorithm is to be prepared to solve different types of job shop problem.

\section{Nomenclature}

$\mathrm{C}_{\max }=$ Makespan, total processing time for the job sequence $\mathrm{T}_{\mathrm{avg}}=$ Average job flow time, it is the total flow time of all jobs divided by the number of jobs.

\section{References}

1. Johnson, S.M., Optimal Two- and Three-Stage Production Schedules with Setup Times Included, Naval Research Logistics, Vol.1, No.1, pp.61-68, 1954.

2. Palmer, D.S., Sequencing Jobs through a Multi-stage Process in the Minimum Total Time - A Quick Method of Obtaining a Near Optimum, Journal of the Operational Research Society, Vol.16, No.1, pp.101-107, 1965.

3. Campbell, H.G., Dudek, R.A., Smith, M.L., A Heuristic Algorithm for the $\mathrm{n}$ Job, m Machine Sequencing Problem, Management Science, Vol.16, No.10, pp.630-637, 1970.

4. Nawaz, M., Enscore Jr, E.E., Ham, I., A Heuristic Algorithm for the $\mathrm{m}$ Machine, $\mathrm{n}$ Job Flow-shop Sequencing Problem, Omega, Vol.1, No.1, pp.91-95, 1983.

5. Man, K.F., Tang, K.S., Kwong, S., Genetic Algorithms: Concepts and Applications, IEEE Transactions on Industrial Electronics, Vol.43, No.5, pp.519-534, 1996.

6. Laha, D., Mandal, P., Improved Heuristically guided Genetic Algorithm for the Flow Shop Scheduling
Problem, International Journal of Services and Operations Management, Vol.3, No.3, pp.316-331, 2007.

7. Li, Y., Chen, Y., A Genetic Algorithm for Job-Shop Scheduling, Journal of Software, Vol.5, No.3, pp. 269274, 2010.

8. Verma, S., Jain, M., Choudhary D., Solving the Job-Shop Scheduling Problem by using Genetic Algorithm, International Journal of Computational Science and Mathematics, Vol.3, No.1, pp.93-98, 2011.

9. Yadav, P.K., Prajapati, N.L., An Overview of Genetic Algorithm and Modeling, International Journal of Scientific and Research Publications, Vol.2, No.9, pp.1-4, 2012.

10. Singhal, E., Singh, S., Dayma A., An Improved Heuristic for Permutation Flow Shop Scheduling (NEH algorithm), International Journal of Computational Engineering Research (IJCER), Vol.2, No.6, pp.95-100, 2012.

11. Semanco, P., Modrak, V., A Comparison of Constructive Heuristics with the Objective of Minimizing Makespan in the Flow-Shop Scheduling Problem, Acta Polytechnica Hungarica, Vol.9, No.5, pp.177-190, 2012.

12. Modrak, V., Semanco, P., Kulpa, W., Performance Measurement of Selected Heuristics Algorithms for Solving Scheduling Problems, $11^{\text {th }}$ International Symposium on Applied Machine Intelligence an Informatics (SAMI), IEEE, pp.205-209, 2013.

13. Choudhari, D., Khanna, R., Flow Shop Scheduling Problems - A Survey, International Journal of Scientific \& Engineering Research, Vol.5, No.12, pp.34-38, 2014.

14. Milosevic, M., Lukic, D., Durdev, M., Antic A., Borojevic, S., An Overview of Genetic Algorithms for Job Shop Scheduling Problems, Journal of Production Engineering, Vol.18, No.2, pp.11-15, 2015.

15. Silva, C., Klement, N., Solving a Multi-periods Job-shop Scheduling Problem using a Generic Decision Support Tool, Procedia Manufacturing, Vol.11, pp.1759-1766, 2017.

16. Liu, W., Jin, Y., Price, M., A New Improved NEH Heuristic for Permutation Flowshop Scheduling Problems, International Journal of Production Economics, Vol.193, pp.21-30, 2017.

\section{Biographical notes}

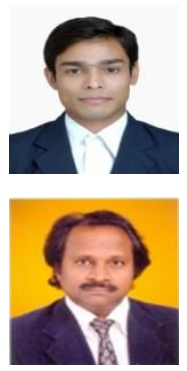

Raushan Kumar has received B.E. in Mechanical Engineering from Chhattisgarh Swami Vivekanand Technical University Bhilai. He is M.Tech. scholar of Department of Mechanical Engineering of Chhatrapati Shivaji Institute of Technology Durg. He is pursuing M.Tech in Industrial Engineering and Management from Chhattisgarh Swami Vivekanand Technical University Bhilai.

Dr.Sridhar has received Ph.D. in Mechanical Engineering from Jawaharlal Nehru Technological University, Hyderabad.. Presently working as professor in the Department of Mechanical Engineering of Chhatrapati Shivaji Institute of Technology Durg. 